\title{
Monitoring of drinking water quality using automated ATP quantification
}

Hansen, C. B.; Kerrouche, A.; Tatari, K.; Rasmussen, A.; Ryan, T.; Summersgill, P.; Desmulliez, M. P.Y.; Bridle, H.; Albrechtsen, Hans-Jørgen

Published in:

Journal of Microbiological Methods

Link to article, DOI:

10.1016/j.mimet.2019.105713

Publication date:

2019

Document Version

Peer reviewed version

Link back to DTU Orbit

Citation (APA):

Hansen, C. B., Kerrouche, A., Tatari, K., Rasmussen, A., Ryan, T., Summersgill, P., Desmulliez, M. P. Y., Bridle, H., \& Albrechtsen, $\mathrm{H}$-J. (2019). Monitoring of drinking water quality using automated ATP quantification. Journal of Microbiological Methods, 165, [105713]. https://doi.org/10.1016/j.mimet.2019.105713

\section{General rights}

Copyright and moral rights for the publications made accessible in the public portal are retained by the authors and/or other copyright owners and it is a condition of accessing publications that users recognise and abide by the legal requirements associated with these rights.

- Users may download and print one copy of any publication from the public portal for the purpose of private study or research.

- You may not further distribute the material or use it for any profit-making activity or commercial gain

- You may freely distribute the URL identifying the publication in the public portal 


\title{
Monitoring of drinking water quality
} using automated ATP quantification

\author{
Hansen, C.B. ${ }^{1}$ Kerrouche, A. ${ }^{2}$, Tatari, K. ${ }^{1}$, , Rasmussen, A. ${ }^{1}$, Ryan, T. $^{3}$, Summersgill, P. ${ }^{3}$, Desmulliez,
} M.P.Y. ${ }^{4}$, Bridle, H. ${ }^{5}$ and Albrechtsen, H.J. ${ }^{1}$

1. Technical University of Denmark, Lygnby, Denmark.

2. Napier University, Edinburgh, Scotland, UK.

3. Epigem, Redcar, UK.

4. Multi-Modal Sensing and Micro-Manipulation Centre (CAPTURE); Institute of Sensors, Signals and Systems (ISSS); Heriot-Watt University, Edinburgh EH14 4AS, Scotland, UK.

5. Institute of Biological Chemistry, Biophysics and Bioengineering; Heriot-Watt University, Edinburgh EH14 4AS, Scotland, UK.

Abstract: A microfluidic based system was developed for automated online method for the rapid detection and monitoring of drinking water contamination utilising microbial Adrenosine-5'Triphosphate (ATP) as a bacterial indicator. The system comprises a polymethyl methacrylate based microfluidic cartridge inserted into an enclosure incorporating the functions of fluid storage and delivery, lysis steps and real-time detection. Design, integration and operation of the resulting automated system are reported, including the lysis method, the design of the mixing circuit, the choices of flow rate, temperature and reagent amount. Calibration curves of both total and free ATP were demonstrated to be highly linear over a range from $2.5-5,000 \mathrm{pg} / \mathrm{mL}$ with the limit of detection being lower than $2.5 \mathrm{pg} / \mathrm{mL}$ of total ATP. The system was trialled in a lab study with different types of water, with lysis efficiency being found to be strongly dependent upon water type. Further development is required before online implementation.

Keywords: microfluidics, drinking water, microbial contamination, monitoring, ATP

\section{Introduction}

Monitoring the quality of drinking water confirms the success of treatment systems, and the integrity of distribution networks, in the delivery of pathogen-free water. Most of the current testing of faecal indicators undertaken by water utilities is lab-based, time consuming and requires culturing, preventing therefore real-time warning of presence of pathogens. To combat these limitations, development of online methods has accelerated, with commercially available systems [1] as well as trial set-ups being adopted in water systems worldwide for source water surveillance [2] and distribution network quality control [3]. Microbial water quality monitoring systems based on optical, electrical or molecular methods of detection have all been developed, though not all have suitability for online or early-warning approaches [4]. For early-warning systems a variety of approaches have 
been tested ranging from the utilisation of online measurements of conductivity and/or turbidity as a proxy [5] to automated culture based systems, online flow cytometry platforms [6], particulate imaging using 3D scanning [7], microbiome analysis [8] and use of indicators such as trypthopan [9] or adenosine-5'-triphosphate (ATP) [10]. ATP was recently found to be the most accurate approach in a comparison of three different sensor types [11].

ATP measurements are widely used in the analysis of microbial contamination in a variety of industries [12], including the water industry [3]. Total ATP in drinking water is the sum of microbial ATP (in active and viable cells) and free ATP. , An ATP assay will be able to detect either total ATP if an extraction reagent is used, or free ATP only without this reagent. The microbial ATP content can therefore be calculated as the difference between these two measurements. Recent work has highlighted the utility of ATP in detecting microbial ingress in drinking water by demonstrating that the approach was more sensitive than total direct counts and more rapid than culture based methods such as heterotrophic plate counts and Coliliert-18 [10]. However, the system was used in a benchtop batch processing setup which is bulky and laboratory based. As a proven fast and reliable technique, online integration of ATP quantification for drinking water quality would be a very useful tool, (e.g. Applitek [13]).

Microfluidics refers to the design, manufacture and testing of systems capable of manipulating and transporting tiny volumes of water with channel dimensions in the order of micrometres. Microfluidic technologies have been applied to numerous biological and biomedical challenges, including water quality monitoring [14]. The advantages of microfluidics are precise, automated control over reagent addition and mixing, an on-chip analysis and measurement approach avoiding human error or contamination and a small, portable device using small quantities of expensive reagents per measurement. Microfluidics has been utilised for ATP detection; specifically, microfluidics-based ATP devices have been reported for submetabolomic analysis [15], detecting airborne microorganisms [16] and for sea water monitoring $[12,17]$. For water quality applications E. coli 0157:H7 detection was reported by integration of the ATP assay with immunomagnetic separation [18] and a paper based microfluidic system was used with Salmonella [19]. Here, we report on the development and optimisation of a microfluidic device, integrated into a detection system, for ATP quantification for drinking water quality applications. The focus of the paper is on the design and operation of the system and the impact of lysis protocol on device performance in lab studies. Additionally, unlike in the previous systems, detection of both total and free ATP has been investigated.

II. Materials and Methods 
69

ATP is most commonly measured by a chemiluminescence assay (Luc assay). The first step of the assay is the extraction of ATP from the cells by lysisreagents (typically cold extractants) $[3,20,21]$. The extracted ATP is then quantified by a two-step reaction that uses the firefly luciferase enzyme (Luc) and its substrate luciferin $\left(\mathrm{D}-\mathrm{LH}_{2}\right)$. The bioluminescence is also dependent on the oxygen $\left(\mathrm{O}_{2}\right)$ concentration and the presence of $\mathrm{Mg}^{2+}$ that leads to the formation of the ATP- $\mathrm{Mg}^{2+}$ complexes as illustrated in reactions 1 and 2 [22].

$\mathrm{LuC}+{ }_{\mathrm{D}-\mathrm{LH}}+\mathrm{ATP}-\mathrm{Mg}^{2+} \leftrightarrow$ Luc $\cdot$ D $-\mathrm{LH}_{2}-\mathrm{AMP}+\mathrm{PPi}-\mathrm{Mg}^{2+}$

(reaction 1)

Where Luc.D.LH 2 -AMP (D-luciferil-adenylate) is the enzyme-bound intermediate generated after the hydrolysis of ATP to AMP (adenosine monophosphate), and PPi- $\mathrm{Mg}^{2+}$ is the inorganic pyrophosphate $\left(\mathrm{P}_{2} \mathrm{O}_{7}^{4}\right)$ bound to $\mathrm{Mg}^{2+}$.

In the second reaction, the intermediate $\mathrm{Luc}_{\mathrm{D}-\mathrm{L}} \mathrm{LH}_{2}$-AMP is oxidized and decarboxylized, emitting light whose intensity is proportional to the initial ATP content [22]:

Luc. D-LH $2-A M P+\mathrm{O}_{2} \leftrightarrow \mathrm{LuC}+\mathrm{AMP}+\mathrm{CO}_{2}+\mathrm{OxyLH}_{2}+\mathrm{hv}$ (reaction 2)

Where $\mathrm{OxyLH}_{2}$ (oxyluciferin) is the light emitting molecule and $h v$ is the energy of the emitted photons whose wavelength lies in the yellow-green spectrum. The light emission reaches its maximum intensity within a second, after which it decreases depending on the assay conditions and the luciferase concentration [23]. Low luciferase concentrations give a relatively stable light emission, while high luciferase concentrations, also known as flash reagents, give light emission that lasts for a very short time. The bioluminescence reaction is particularly sensitive to $\mathrm{pH}$ and temperature. The optimal reaction temperature is $25^{\circ} \mathrm{C}$ [24] whilst the optimal pH during the assay is between 6 and 8 [23] and is controlled by buffer addition. The luciferin-luciferase reagents were the LuminATE kit (92687, Celsis).

\section{Microfluidic Design, Microfabrication and Operation:}

In similarity to ATP detecting microfluidic systems created by Lee [16] and Fukuba [12] the device is composed of a mixing zone for the various liquids, a detection area and several inlets to introduce the sample, lysis agent and detection reagents. More specifically, from left to right in Figure 1, the sample and reagents are introduced from their storage flasks to inlet channels in the microfluidic system via semi-rigid tubing (Cheminert ${ }^{\circledR}$ ). Two sequential mixing sections made of serpentine channels ensure the efficient combination of the sample with the lysis reagent (liquids from inlets 1 and 2), and of the lysed sample with the chemiluminescence reagents (output of first mixing section with liquid from 
inlet 3). The reagents require storage at $4^{\circ} \mathrm{C}$ degrees to preserve their reactivity and shelf life. The long serpentine channels with the microfluidic device have therefore also been designed to allow time for the liquids to reach the optimal reaction temperature for the Luc reaction. Finally, the sample enters a detection zonein which the channel forms a $32 \mathrm{~mm}$ diameter spiral. The spiral shape ensures maximum capture of the luminescent signal from the samples. To ensure the produced light is reflected towards the detector a metallic layer of foil was placed externally below the device and opposite the spiral. Two different mixing designs were trialled and assessed via imaging (Dinolite) of the mixing of food dye.

The microfluidic system was manufactured by Epigem Ltd using acrylic (PMMA) and epoxy. The choice of materials was based on the requirement for long term robustness, good compatibility with wet conditions in the device and good light transmission in 550-650 nm wavelength produced by the Luc reaction.

Sample and reagents were supplied to the microfluidic device inlets using a peristaltic pump (ISMATEC Reglo ICC). After calibration, the pump displayed a maximum 5\% deviation from the nominal flow rate. The $0.51 \mathrm{~mm}$ inner diameter pumping tube used for the peristaltic pumps was made from a silicone based polymer and was linked to a $0.2 \mathrm{~mm}$ inner diameter and $1.59 \mathrm{~mm}$ outer diameter small bore analytical semi-rigid tubing (Cheminert) to minimise the volume of expensive reagents used ( $31 \mu \mathrm{L}$ per meter of tubing). Further reduction of volume was carried out using short length connections from the pumping tube to the reagents and sampling reservoirs next to the pump as well as the microfluidic inlets. Two-way pinch valves with an internal volume of $14 \mu \mathrm{L}$ (NI USB 6525 from National Instruments), were selected to control the flow of reagents and solution. and are electrically actuated using controllable solid state relays.

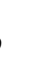

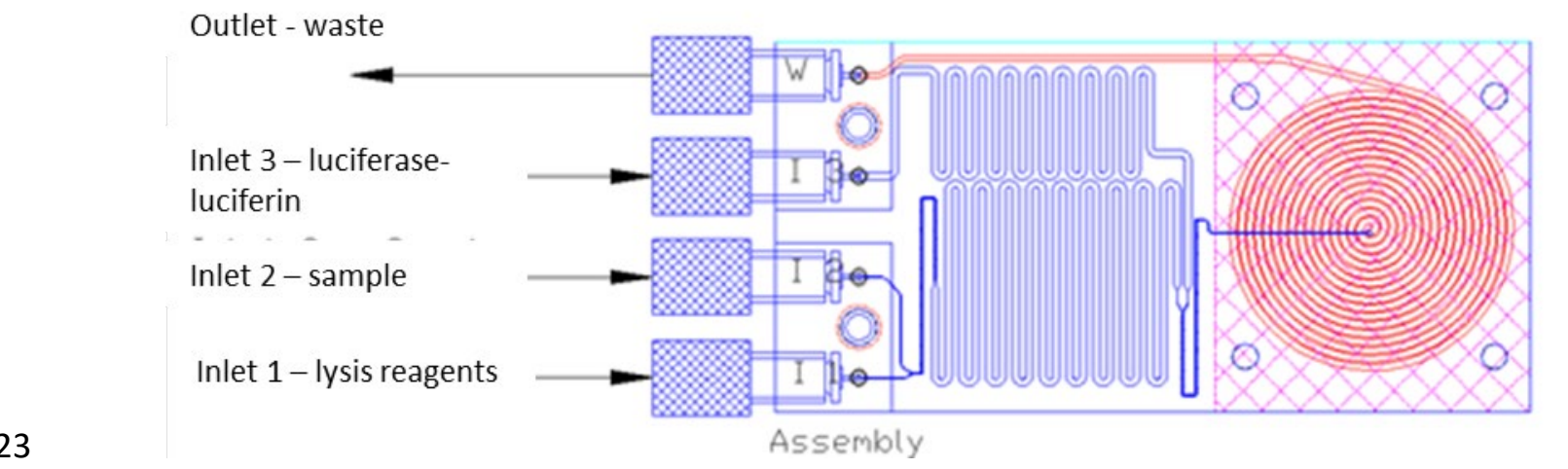

Figure 1: Overview of the design and operation of the microfluidic system. 
127 This mixing and detection system, whose schematic is displayed in Figure 2, is contained within a light128 tight box. The box is thermally insulated and includes a temperature control system for constant temperature of $25^{\circ} \mathrm{C}$ to ensure stable and optimal temperature condition for the Luc reaction. A small fan is also employed to avoid any gradient of temperature inside the box. The temperature of an aluminium plate is regulated using an electrical heater fitted on the underside of the plate. The temperature of the plate is measured by a K-type thermocouple sensor on the side of the plate and the signal is used by a PID controller to maintain the desired temperature. The precision of the regulating loop is within $\pm 0.1^{\circ} \mathrm{C}$, and the temperature can be set in the interval from the surrounding temperature of the detection box up to $36^{\circ} \mathrm{C}$. A $3 \mathrm{~mm}$ thick, $98 \%$ total light transmission, antireflection coated glass was inserted between the head of the Photo-Multiplier Tube (PMT) and the microfluidic device to avoid the heating up of the light sensitive coating of the detector.

A H9319-01 Hamamatsu PMT detector was selected as it offers very effective in-built amplification of low light intensity signals for easier detection, the right spectral response (550 nm-650 nm) of the photosensitive material inside the detector as well as a low level of thermal noise. The spiral readout area on the microfluidic device was made slightly larger, with a diameter of $\varnothing 32 \mathrm{~mm}$, to ensure full use of the $\varnothing 22 \mathrm{~mm}$ diameter detector area rather than trying to minimize the volume of the spiral.

The PMT detector and the centre of the spiral are placed on the same longitudinal axis, with the PMT mounted in the lid of the assay box as shown in Figure 2. The tubing and the electrical wires enter the box through tight rubber gaskets that prevent light from entering the detection box.

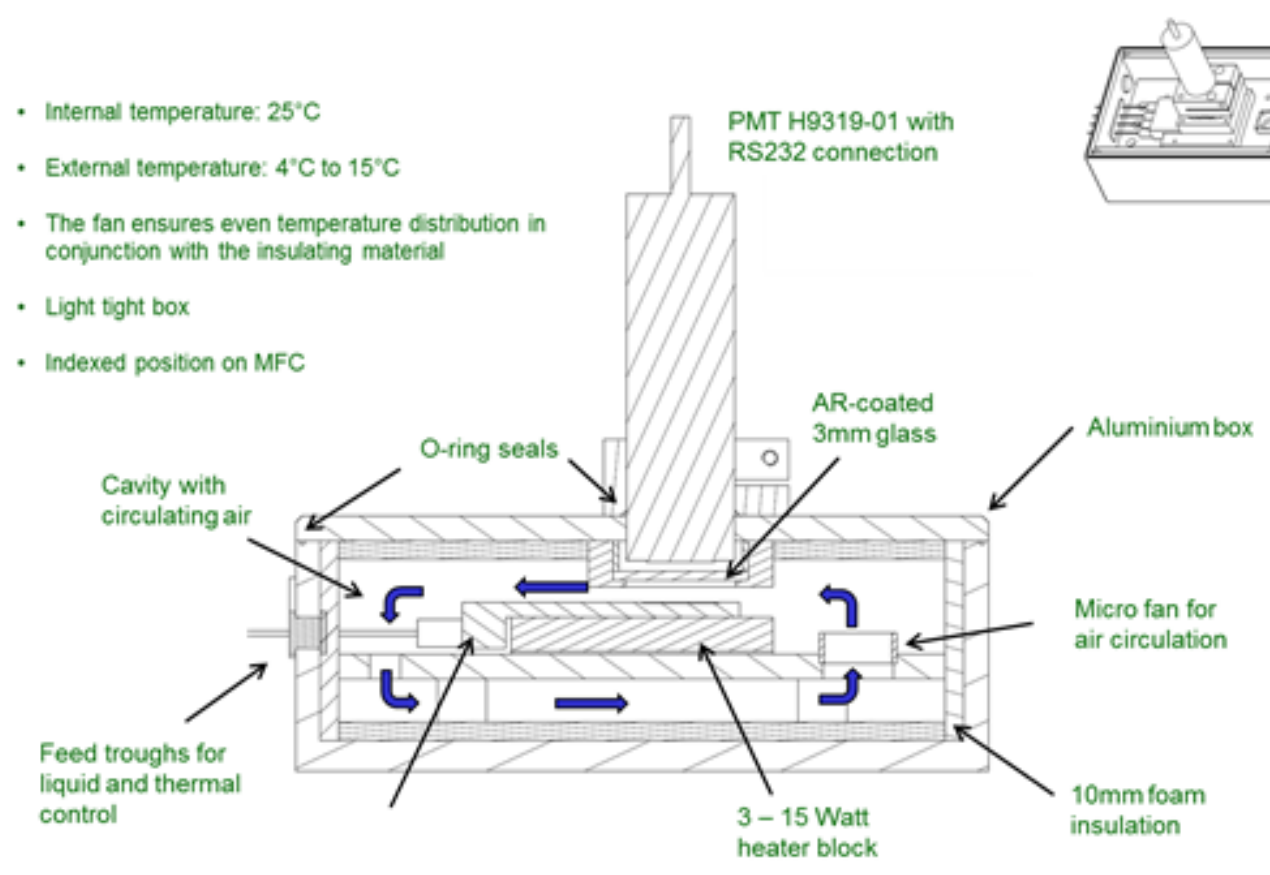


System Evaluation:

ATP standard salt (92638, Celsis) was reconstituted in Lumin (PM) buffer (92678, Celsis), MilliQ-water or sterile filtrated autoclaved drinking water at a concentration of $1 \times 10^{6} \mathrm{pg} \mathrm{ATP} / \mathrm{mL}$ and stored at $1^{\circ} \mathrm{C}$. Dilutions were then made using autoclaved, sterile filtered tap water (i.e. ATP free) for standards experiments in the range $2.5-500 \mathrm{pg} / \mathrm{mL}$. Subsequently, the system was tested with different water types (collected at DTU). The wastewater utilised was diluted 100 times before use. The rainwater sample was collected after storage in a tank for 3 weeks. The tap water sample was taken from the lab tap during the middle of the day, and after a preliminary measurement where low levels of ATP were measured the ATP standard salt was used to spike the sample for the study evaluating different lysis approaches.

159

III. Results and Discussion

\section{A. ATP Detection and Calibration}

Using a $1000 \mathrm{pg} / \mathrm{mL}$ ATP standard, the impact of operating conditions on the obtained optical signal was investigated with flow rates ranging from $50 \mu \mathrm{l} / \mathrm{min}$ to $300 \mu \mathrm{L} / \mathrm{min}$ and temperatures between $25^{\circ} \mathrm{C}$ and $32^{\circ} \mathrm{C}$. As shown in Figure $3 \mathrm{~A}$, flow rates within the studied range have no impact upon the output signal whereas temperatures above $28^{\circ} \mathrm{C}$ result in a small decrease in the signal with results being similar between $25^{\circ} \mathrm{C}$ and $27^{\circ} \mathrm{C}$ (Figure 3B). The optimal temperature of $25^{\circ} \mathrm{C}$ for the Luc reaction is also confirmed. Different ratios of sample to luciferin-luciferase reagents were also tested, as shown in Figure 3C, to explore the possibility of reducing the amount of enzyme required for detection. Unsurprisingly, Figure $3 \mathrm{C}$ shows that the greater amount of reagent is employed, the higher the obtained signal. From these three results, operating conditions of $100 \mu \mathrm{L} / \mathrm{min}, 25^{\circ} \mathrm{C}$ and a $1: 1$ ratio of sample/reagent were selected.

Calibration curves for the PMT detector were obtained using the optimised detection parameters described above for both total and free ATP detection as well as determination of the limit of detection. These curves are essential to convert the signals expressed in Relative Light Units (RLU ) into $\mathrm{pg} / \mathrm{mL}$ concentrations and to account for any possible inhibition or quenching caused by the lysis reagent. Two series of nine standards in the $2.5-500 \mathrm{pg} / \mathrm{mL}$ ATP concentration range were run to obtain the calibration curves for total and free ATP as shown in Figure 4. Indicative peak responses of 
the standards and peak decrease back to the background levels after the injection of the standards are shown in the Supplementary Information (Figure S2). The two calibration curves fitted well into a linear model ( $R^{2}=0.997$ for total and $R^{2}=1$ for free ATP). The lowest standard, $2.5 \mathrm{pg} / \mathrm{mL}$, is within the noise for the total ATP calibration curve and provides a signal to noise ratio (SNR) of 1.5:1 for the free ATP calibration curve. This result indicates that the limit of detection is better than $2.5 \mathrm{pg} / \mathrm{mL}$, a slight improvement over the previously reported microfluidic ATP analyser [12]. This is also significantly lower than the paper based device reported in 2015 although the paper system did not use a PMT detector [19].
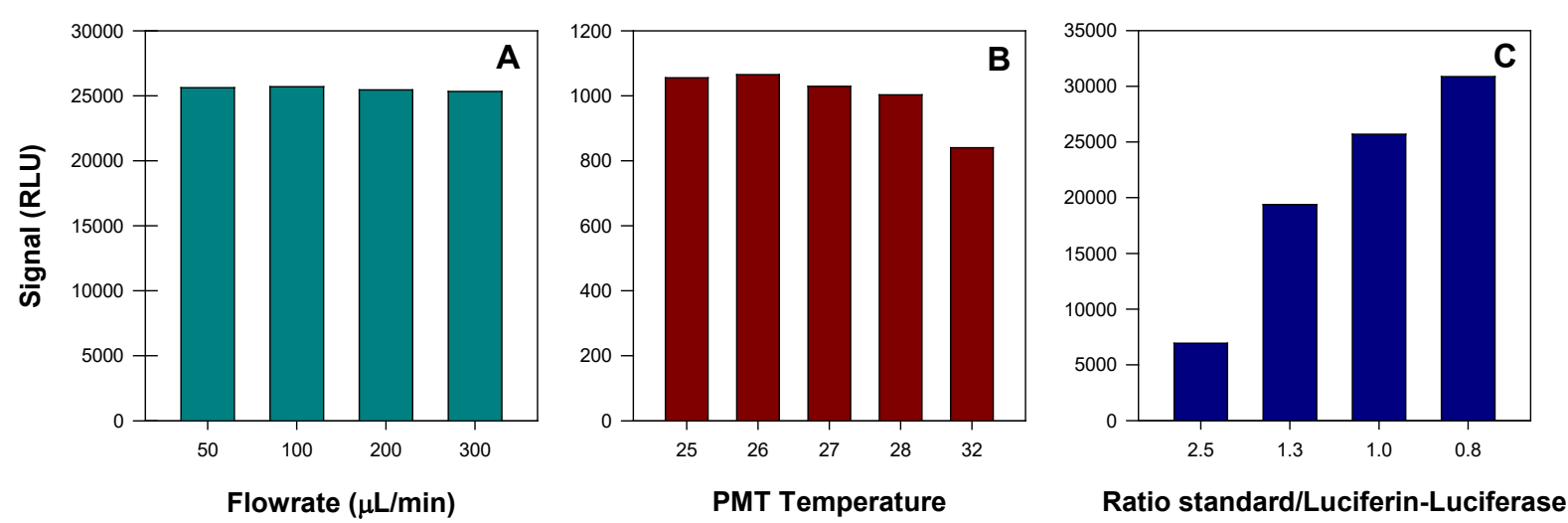

Figure 3: Characterisation of operational parameters for detection expressed as Relative Light Units (RLU). A) Impact of varying flow rates. B) Effect of varying temperatures in the PMT stage. C) Influence on the output signal of the luciferin-luciferase reagent for detection.
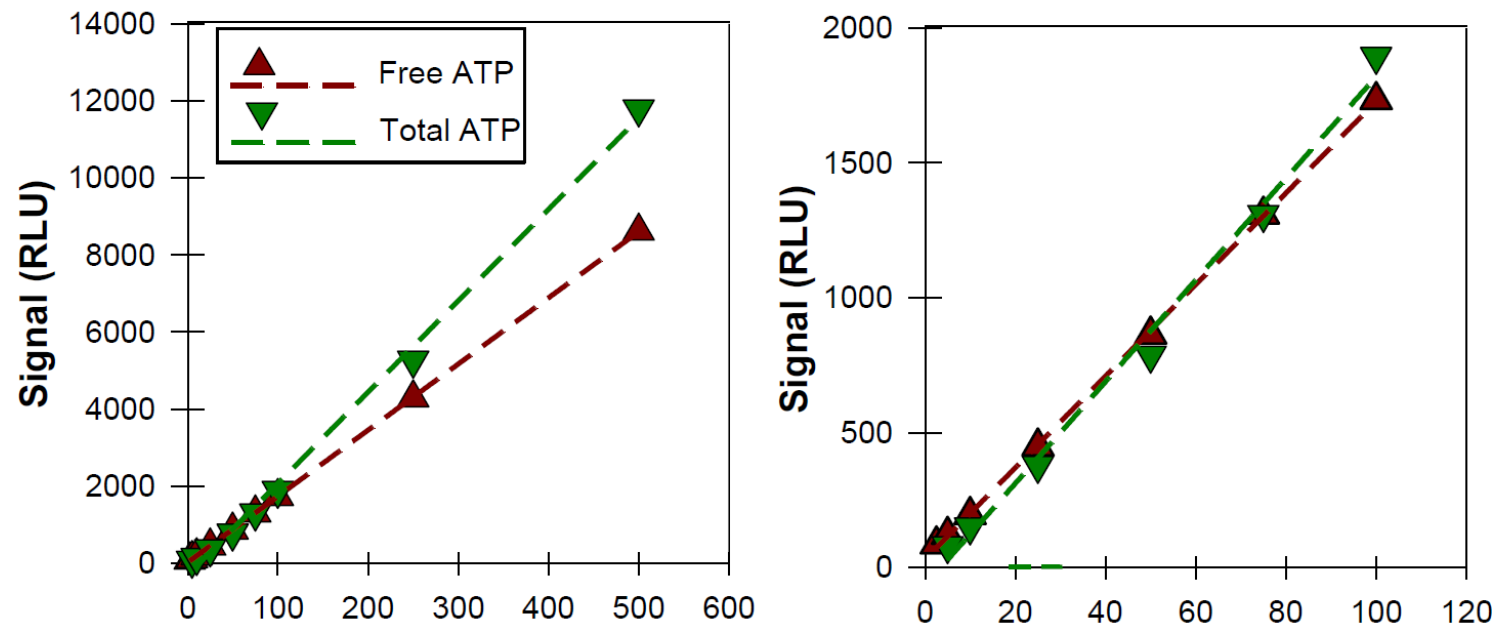

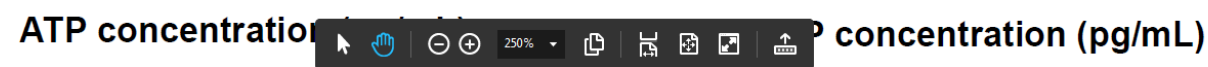

Figure 4: Calibration curves for both total and free ATP. Figure on the left shows the entire investigated range whereas the figure on the right is a magnified view of the lower end of the concentration range.

B. Optimisation of the Sample Preparation Procedure 
Sample processing is often the most challenging part of a monitoring solution. For our device, two critical tasks were considered: the efficacy of the mixing of the reagents with the sample and the efficiency of the lysis process.

201

Fluid flows are generally laminar in microfluidic devices and mixing occurs primarily by diffusion, which tends to occur over long distances. Therefore mixing can be enhanced by splitting and laminating the flow or by introducing recirculating flows, thereby effectively reducing the diffusion length [25]. Two configurations of mixers were investigated as shown in Figure 5: the serpentine mixer consisting of 161 meandering turns and the Tesla mixer [26]. The mixing efficiency was investigated in the serpentine mixer by observing under a microscope the mixing of a dark liquid dye and a clear liquid as shown in Figure 5B. From this figure, the distances $L_{1}$ and $L_{2}$ are defined as the width of each of the two liquids within the channel, when it is possible to discern the boundary from imaging The mixing efficiency can therefore be calculated using the equation $\eta=\left(? ?_{1}-? ?_{2}\right) / ?_{1}$ with $L 1$ and L2 indicated in Figure 5B. The mixing efficiency was plotted as a function of distance from the mixer inlet for 5 different flow rates in the $10-50 \mu \mathrm{L} / \mathrm{min}$ range (Figure $5 \mathrm{C}$ and D). As it was not possible to distinguish the mixed and unmixed zones above $70 \%$ only the first part of the mixer structure was examined (35 $\mathrm{mm}$ of length for the serpentine design). The Tesla mixer design displayed an increased mixing efficiency compared with the serpentine, reaching a 70\% mixing efficiency after just a $15 \mathrm{~mm}$ distance from the inlet at high flow rates. An additional advantage is the lower fluidic resistance in the channel (Figure $5 \mathrm{C}$ and $\mathrm{D}$ ). Note that the mixing efficiency increases for higher flow rates for both mixing designs. The mixing distance required is shorter than that reported for the seawater ATP analyser, which utilises a serpentine channel length of $133 \mathrm{~mm}$ for the mixing of lysis reagent and sample at the end of which the luminescence reagents are added and then allows a straight channel distance of $350 \mathrm{~mm}$ until the bioluminescence is measured. The mixing in a straight channel is ascribed to the asynchronous operation of their peristaltic pumps causing pulsation [17]. Shorter mixing reduces device footprint. 

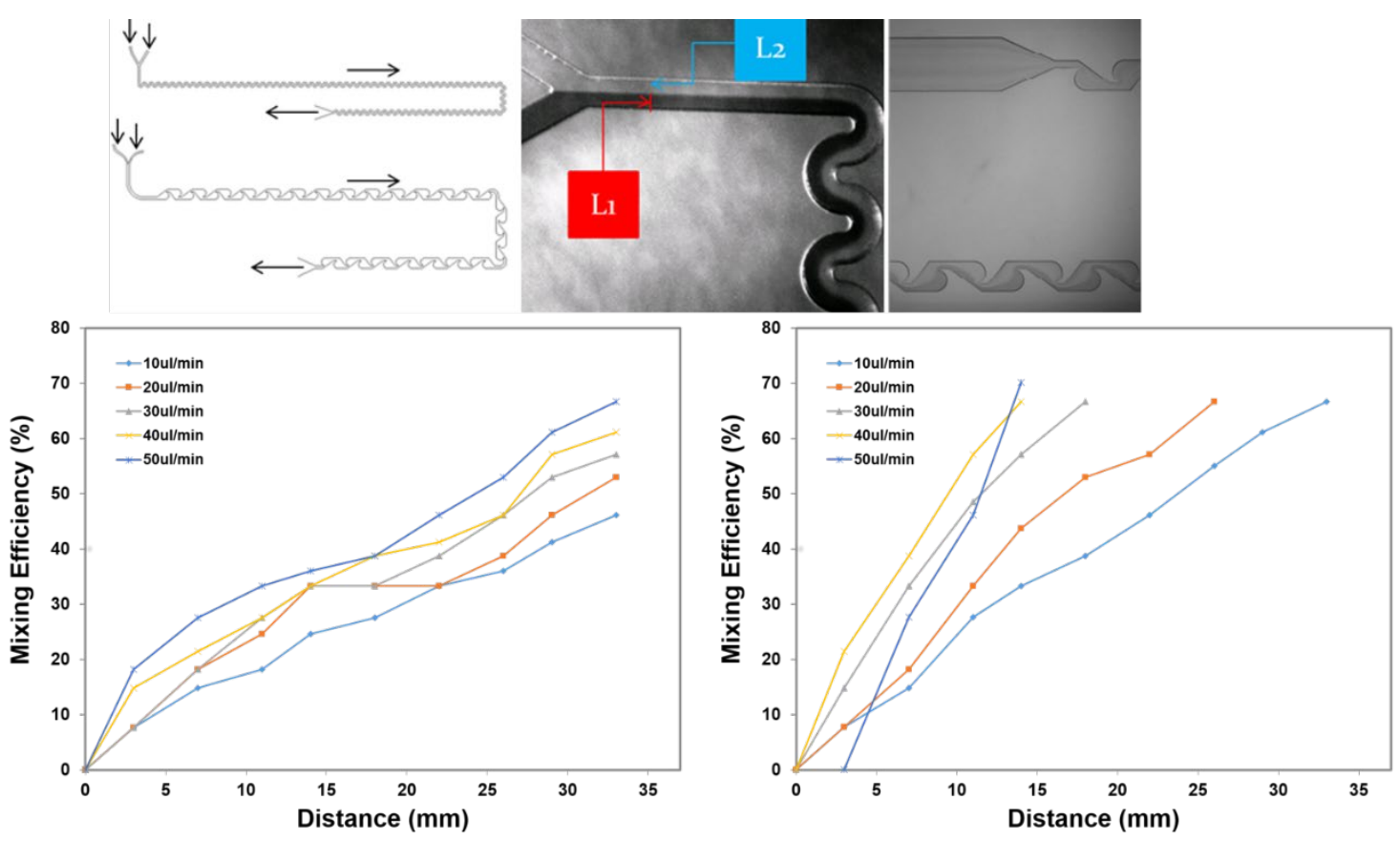

Figure 5. A) Top left - Design of the two different mixing channel structures (top -serpentine; bottom - Tesla). B) Top right - Images from the mixing experiments. C) Bottom left - Mixing efficiency for the serpentine design. D) Bottom right - Mixing efficiency for the Tesla layout.

\section{Device Performance with Varying Types of Water}

Further work was undertaken to quantify the total and free ATP in a variety of water types. The performance of two different lysis approaches was also carried out with these water types as shown in Figure 6. The previously generated calibration curve was utilised to convert signal into ATP concentration, although this does not account for any impact of the water type on ATP measurement performance. While chemical lysis was shown to provide good output signals, as shown in Figure 6, problems were observed with channel clogging due to the lysis agent interacting with the luciferinluciferase reagents (Figure S3). Fukuba et al employed EDTA to avoid issues of precipitation [17]. As an alternative, thermal lysis was incorporated into the system and trialled. Preliminary data for a calibration standard of $100 \mathrm{pg} / \mathrm{mL}$ suggests that no damage or destruction of the ATP has occurred during the thermal lysis step. Moreover similar RLU as the chemical lysis experiments is demonstrated, with measurements of free (without lysis) and total (with lysis) ATP almost identical (Figure S4). This data also indicates that thermal lysis is successful since the signal for total ATP was higher than the signal for free ATP in the $E$. coli suspension and the tap water, meaning that the thermal lysis extracted ATP from the cells in these samples.

Free and total ATP signals were however also similar for the diluted wastewater sample (Figure S4). This result suggests that either thermal lysis did not successfully extract ATP from the cells, or that the 
extracted ATP was consumed before the analysis, due to either the different water matrix or the different types of microorganisms present in each sample. For surface water, rainwater and tap water total ATP was consistently higher than free ATP as shown in Figure 6, suggesting the thermal lysis efficiency problem was specific to wastewater (Figure S4) and requires further investigation in relation to the components in the wastewater. For surface (lake) water, thermal and chemical lysis were equally effective whereas the tap water sample showed variation between Figure 6 and Figure S4 in terms of the effectiveness of thermal lysis. However, when analysing rainwater samples, chemical lysis outperformed the thermal approach in terms of the level of total ATP measured. The rainwater ATP were high though the sample was stored in a collection tank for 3 weeks before processing, and so this data could indicate possible contamination of the storage tank. An E. coli dilution, included as a control, showed the opposite trend whereby thermal lysis gave a much higher detection of total ATP. This data confirms that sample preparation steps are challenging to optimise for waterborne pathogen detection and that universal approaches, for all pathogens and all water types, can be difficult to develop. Other microfluidic approaches to ATP detection have used either chemical or thermal lysis and have not compared the performance. A paper based system for Salmonella detection utilised boiling of the sample as the sample processing steps were undertaken external to the device; this system is cheap and disposable but not suitable for online early warning applications [19]. Otherwise, the microfluidics systems reported have utilised chemical lysis. Potentially problems relating to the interaction of the lysis reagents and particle formation were avoided, even in wastewater samples, in iFAST system due to the use of magnetic beads for specific pathogen capture and washing [18].

267

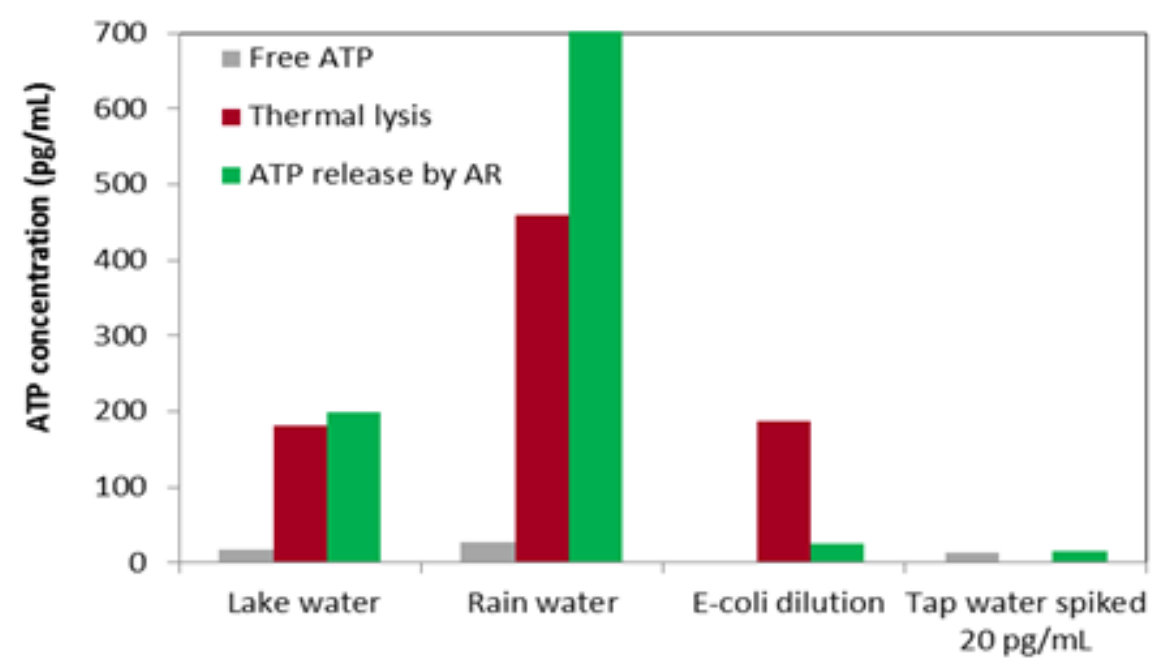


Figure 6. ATP released from the cells by chemical and thermal lysis methods in different types of water. Free ATP is the ATP measured without any releasing step.

\section{Conclusions}

This article presented the development of a microfluidic system acting as an online early warning system for measuring microbial contamination in drinking water samples through quantification of ATP. In terms of detection, system operation with regards to flow rate, temperature and reagent amount were optimised. Highly linear calibration curves for both total and free ATP were determined, estimating the limit of detection to be better than $2.5 \mathrm{pg} / \mathrm{mL}$; a result which is comparable to benchtop studies and one previously reported microfluidic set-up.

For the sample preparation part of the platform, mixing design was optimised with a Tesla layout shown to provide a high mixing efficiency over a short channel length with low fluidic resistance. Different lysis protocols were trialled and efficiency was shown to depend upon the type of water sample.

Overall the developed prototype system demonstrates highly sensitive rapid detection of bacterial contamination in drinking water samples. Future work should concentrate on further investigating the impact of water type on lysis efficiency and consideration of the potential to link with immunomagnetic separation to provide microorganism specific information as well as online testing of the system within drinking water networks.

\section{Supplementary Material}

See supplementary material for further images of the system (Figure S1), the raw data time course for calibration (Figure S2), the clogging observed with chemical lysis (Figure S3) and the preliminary thermal lysis data (Figure S4).

\section{Acknowledgements}

All authors of this article acknowledge the financial support of the European Union through the FP7Integrated Project entitled "Aquavalens” (grant number 311846, theme KBBE-2012-2.5-01).

\section{References}

1. Storey, M.V., B. van der Gaag, and B.P. Burns, Advances in on-line drinking water quality monitoring and early warning systems. Water Research, 2011. 45(2): p. 741-747. 
2. Tryland, I., et al., On-Line Monitoring of Escherichia coli in Raw Water at Oset Drinking Water Treatment Plant, Oslo (Norway). International Journal of Environmental Research and Public Health, 2015. 12(2): p. 1788-1802.

3. Delahaye, E., et al., An ATP-based method for monitoring the microbiological drinking water quality in a distribution network. Water Research, 2003. 37(15): p. 3689-3696.

4. Bridle, H., B. Miller, and M.P.Y. Desmulliez, Application of microfluidics in waterborne pathogen monitoring: A review. Water Research, 2014. 55: p. 256-271.

5. Banna, M.H., et al., Online Drinking Water Quality Monitoring: Review on Available and Emerging Technologies. Critical Reviews in Environmental Science and Technology, 2014. 44(12): p. 1370-1421.

6. Buysschaert, B., et al., Online flow cytometric monitoring of microbial water quality in a fullscale water treatment plant. npj Clean Water, 2018. 1(1): p. 16.

7. Hojris, B., et al., A novel, optical, on-line bacteria sensor for monitoring drinking water quality. Scientific Reports, 2016. 6: p. 23935.

8. Pinto AJ, et al., Spatial-temporal survey and occupancy-abundance modeling to predict bacterial community dynamics in the drinking water microbiome. mBio, 2014. 53(3): p. e01135-14.

9. Simões, J. and T. Dong, Continuous and Real-Time Detection of Drinking-Water Pathogens with a Low-Cost Fluorescent Optofluidic Sensor. Sensors (Basel, Switzerland), 2018. 18(7): p. 2210.

10. Vang, Ó.K., et al., Evaluation of ATP measurements to detect microbial ingress by wastewater and surface water in drinking water, in Water Research. 2014. p. 309-320.

11. Sherchan, S., et al., Near Real-Time Detection of E. coli in Reclaimed Water. Sensors 2018. 18: p. 2303.

12. Fukuba, T., et al., A microfluidic in situ analyzer for ATP quantification in ocean environments. Lab on a Chip, 2011. 11(20): p. 3508-3515.

13. https://www.applitek.com/products/ez-atp.

14. Bridle, H., et al., Detection of Cryptosporidium in miniaturised fluidic devices. Water Research, 2012. 46(6): p. 1641-1661.

15. Liu, B.-F., et al., Microfluidic Chip toward Cellular ATP and ATP-Conjugated Metabolic Analysis with Bioluminescence Detection. Analytical Chemistry, 2005. 77(2): p. 573-578.

16. Lee, S.J., et al. Microfluidic ATP-Bioluminesecence Sensor for Detection of Airborne Microbe. in TRANSDUCERS 2007 - 2007 International Solid-State Sensors, Actuators and Microsystems Conference. 2007.

17. Fukuba, T., et al., Adenosine Triphosphate Measurement in Deep Sea Using a Microfluidic Device. Micromachines, 2018. 9(8): p. 370.

18. Ngamsom, B., et al., A Microfluidic Device for Rapid Screening of E. coli O157:H7 Based on IFAST and ATP Bioluminescence Assay for Water Analysis. Chemistry - A European Journal, 2017. 23(52): p. 12754-12757.

19. Jin, S.-Q., et al., A cost-effective Z-folding controlled liquid handling microfluidic paper analysis device for pathogen detection via ATP quantification. Biosensors and Bioelectronics, 2015. 63: p. 379-383.

20. Hammes, F., et al., Measurement and interpretation of microbial adenosine tri-phosphate (ATP) in aquatic environments. Water Research, 2010. 44(13): p. 3915-3923.

21. Vang, O.K., et al., Evaluation of ATP measurements to detect microbial ingress by wastewater and surface water in drinking water. Water Research, 2014. 64: p. 309-320.

22. Vang, O.K., ATP measurements for monitoring microbial drinking water quality. 2013, Kgs. Lyngby: Technical University of Denmark.

23. Guardigli, M., A. Lundin, and A. Roda, "Classical" Applications of Chemiluminescence and Bioluminescence. Chemiluminescence and Bioluminescence: Past, Present and Future, ed. A. 
Roda. 2011: Royal Soc Chemistry, Thomas Graham House, Science Park, Cambridge Cb4 4wf, Cambs, Uk. 143-190.

24. Ford, S.R. and F.R. Leach, Bioluminescence methods and protocols, in Improvements in the application of firefly luciferase assays. 1998, R. A. Larossa, Humana Press Inc.: Totowa, NJ.

25. Lee, C.-Y., et al., Microfluidic Mixing: A Review. International Journal of Molecular Sciences, 2011. 12(5): p. 3263-3287.

26. Hong, C.-C., J.-W. Choi, and C.H. Ahn, A novel in-plane passive microfluidic mixer with modified Tesla structures. Lab on a Chip, 2004. 4(2): p. 109-113. 

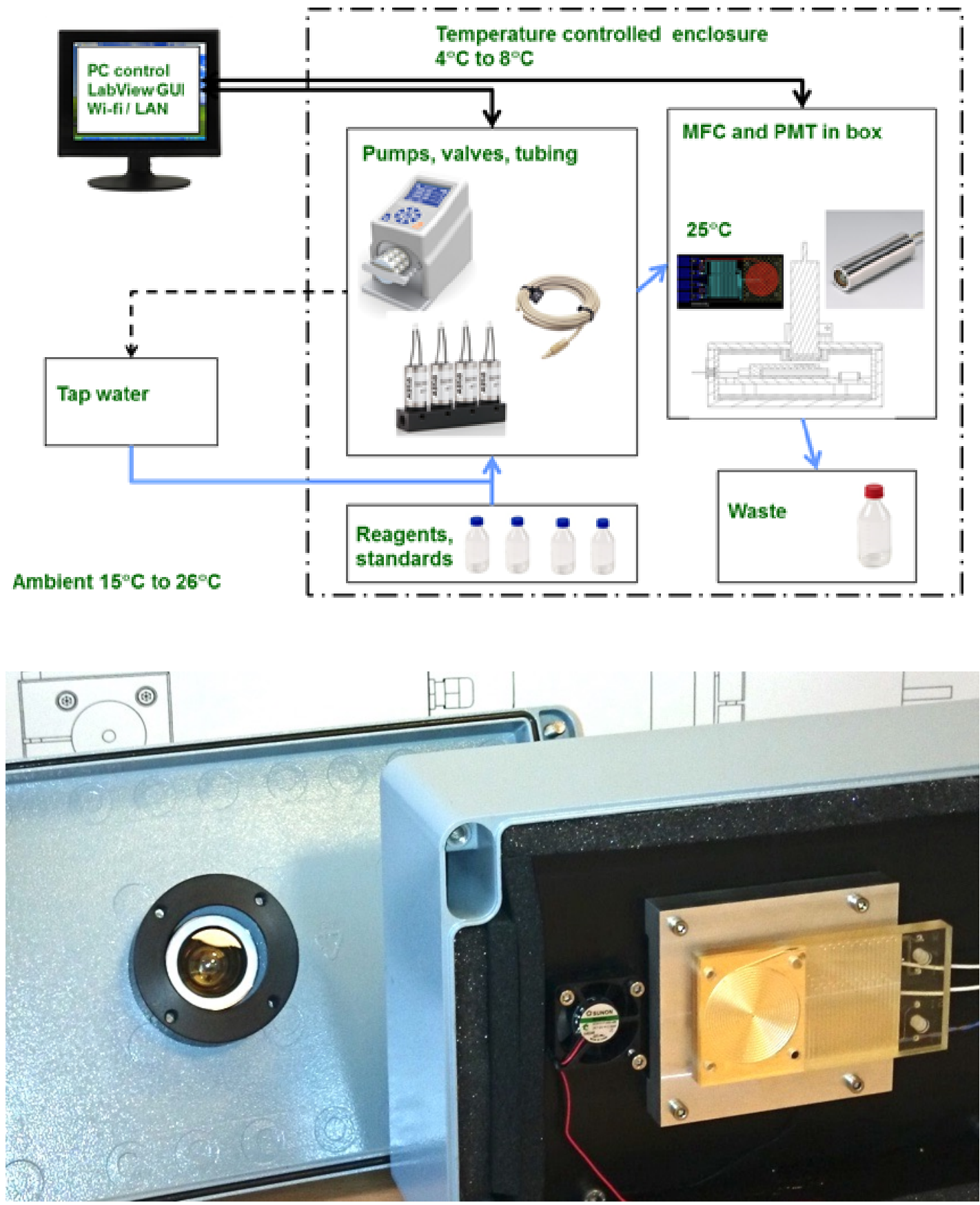

Figure S1

Upper image: A functional schematic of the concept of the ATP platform. A local computer controls the instrumentation needed to configure and perform the online ATP measurements. The instrumentation consists of programmable pumps, controllable valves and a PMT detector assembly that includes the microfluidic based assay. A dedicated graphical user interface (GUI), based on LabView $^{\circledast}$, allows access to work with the system. Data transfer requires connectivity to the internet using either a WiFi- or LAN connection. Fluidic connections for liquids are shown using blue arrows. The temperature controlled enclosure ensures the stability of reagents regardless of local conditions and seasonal variations in ambient temperatures. The measurements are performed on the 
389

390

391

392

393

394

395

396

397

microfluidic cartridge using a highly sensitive photomultiplier tube both mounted in a box in order to exclude external light.

Lower image: The head of the PMT is fixed in the lid on the left side of the picture. The MFC is placed on the temperature controlled aluminium plate in the right side of the picture. The fan visible next to the aluminium plate is used to recirculate the air to maintain a constant temperature throughout the interior of box. Notice the black foam insulation at the rim of the box providing thermal insulation.

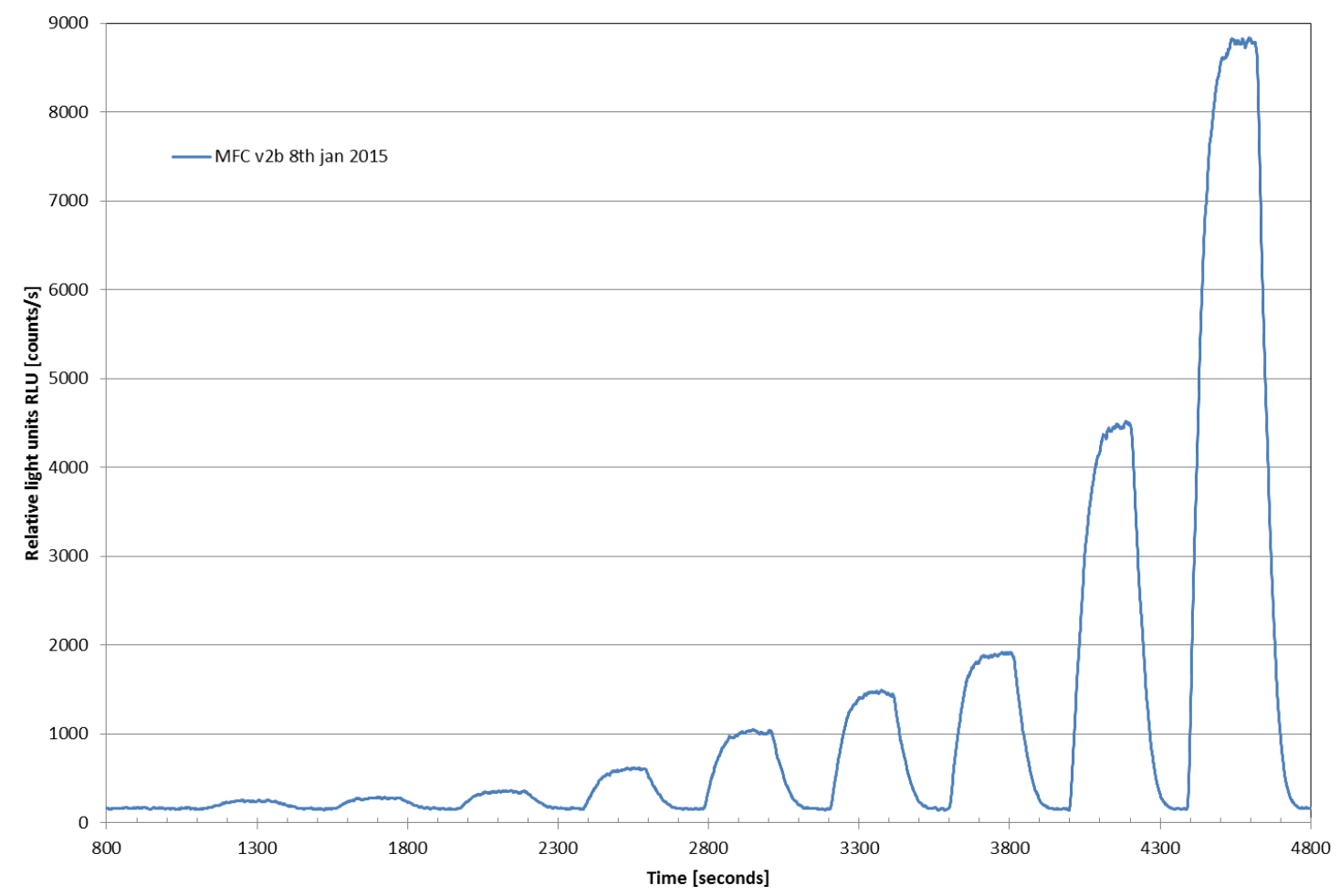

Figure S2

Raw data obtained with ATP-spiked samples using the following concentrations measured in $\mathrm{pg} / \mathrm{mL}$ (left to right) 2.5, 5, 10, 25, 50, 75, 100, 250, 500. 

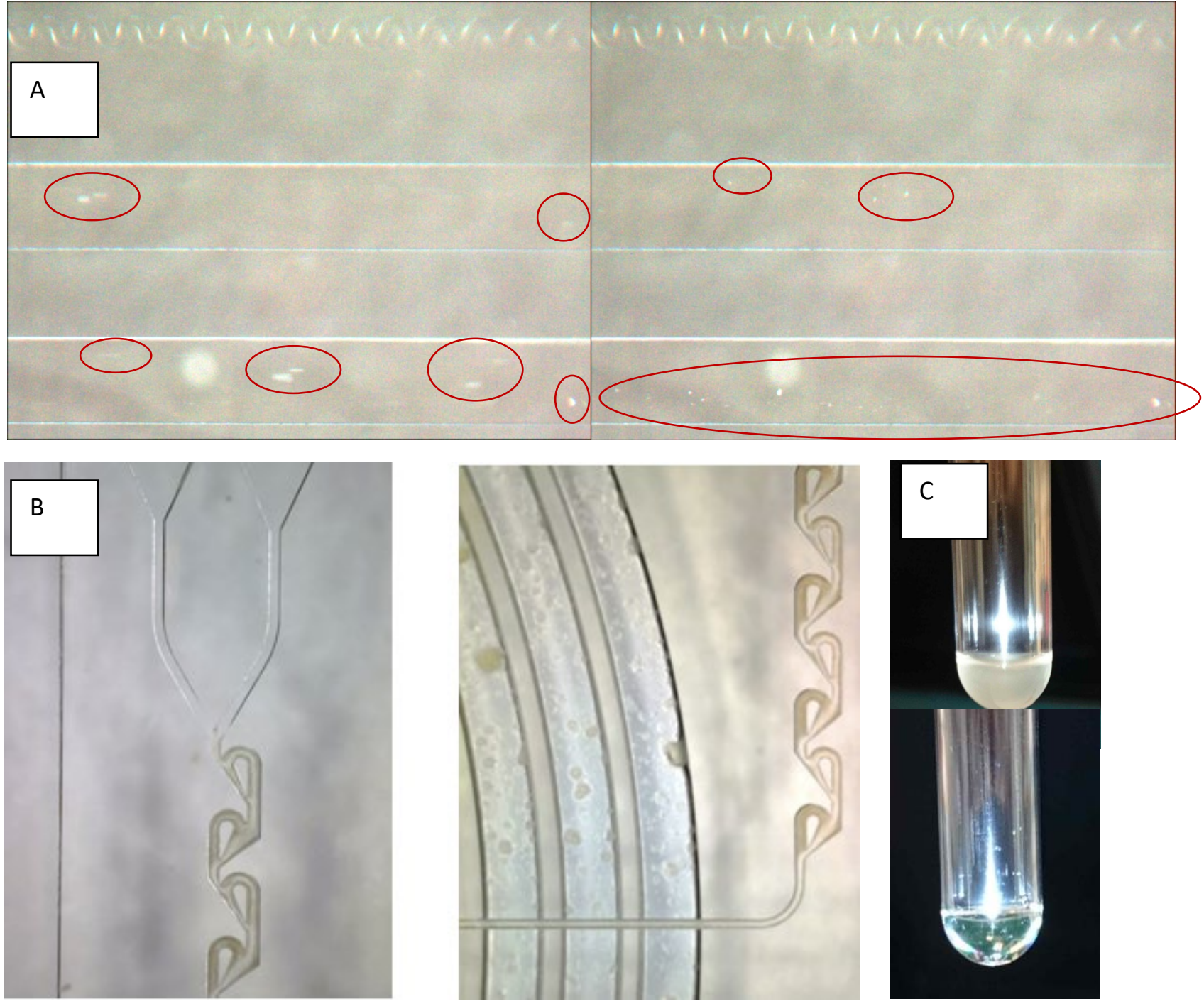

400

401

Figure S3

A: Formation of particles in the microfluidic when ATP free water, lysis reagent and LL are supplied in the three inlet channels. Left panel shows that particles are moving when the flow is on and right panel shows that these particles deposit in the channels when the flow is turned off. The red circles highlight the areas where particles are observed.

B: The left photo shows two clear liquids merging with particulate matter developing downstream in the Tesla mixer structure. On the right picture solid matter can be seen to deposit in the slower 
flowing regions of the mixer. In the spiral readout region larger agglomerates of the solid matter has

415 formed while flowing downstream.

416 C: Formation of particles when mixing ATP free water, lysis reagent and LL in a cuvette results in a 417 turbid liquid (left picture). Picture is taken immediately after mixing the reagents. Mixing of LL with 418 the ATP free water in a cuvette does not generate particles, but the mixture remains clear (right 419 picture).

Figure S4. Preliminary investigations of the performance of thermal lysis. The wastewater was sourced at DTU and diluted 100 times before use in the device. 